\title{
Reflexões Sobre o Papel da Educação Estatística na Formação de Professores no Contexto da Pandemia da Covid-191
}

\section{Reflections on the Role of Statistical Education in Teacher Education in the Context of the Covid-19 Pandemic}

\author{
Suzi Samá*a; Irene Cazorla ${ }^{\mathrm{b}}$, Luciane Velasque ${ }^{\mathrm{c}}$, Leandro Diniz ${ }^{\mathrm{d}}$; Leandro Nascimento ${ }^{\mathrm{e}}$
}

\begin{abstract}
Resumo
A Pandemia da Covid-19 tem gerado dados que a todo momento são divulgados pelas mídias. Este cenário impõe aos professores o desafio de estimular o desenvolvimento de uma atitude crítica do estudante em relação às informações difundidas. Ao trabalhar os conceitos matemáticos e estatísticos envolvidos nesta situação pandêmica, o professor pode instituir novos modos de promover a aprendizagem, a interação e o compartilhamento de significados entre os estudantes. Desse modo, este artigo tem por objetivo refletir sobre como os professores podem trabalhar conceitos matemáticos e estatísticos com os estudantes da Educação Básica no contexto da pandemia. Como suporte teórico, nos ancoramos na Base do Conhecimento proposta por Shulman, focando o conhecimento específico de Estatística, o conhecimento pedagógico e o conhecimento curricular. Apresentamos seis Casos de Ensino construídos a partir dos dados e informações estatísticas disponíveis em sites oficiais, visando dar sentido e significado às informações. Para tal, seguimos as premissas do Letramento Estatístico, dos níveis de leitura e interpretação de gráficos e do senso crítico. Por meio destes casos apresentamos ao professor diferentes recursos, como materiais concretos, simulação por meio de applets do Geogebra, indicadores como letalidade, taxa de mortalidade e prevalência a partir do contexto da pandemia da Covid-19. Salientamos, nesta perspectiva, a importância do conhecimento do contexto e do desenvolvimento da capacidade de formular questões críticas para, assim, contribuir com a formação de professores de forma que estes auxiliem os estudantes a entender a pandemia da Covid-19 e tomar decisões que preservem suas vidas e das pessoas de sua comunidade.
\end{abstract}

Palavras-chave: Educação Estatística. Formação de Professores. Base do Conhecimento. Casos de Ensino. Pandemia da Covid-19.

\begin{abstract}
The Covid-19 Pandemic has generated data that is currently disseminated by the media. This scenario imposes on teachers the challenge of stimulating the students' development for a critical attitude in relation to the information disseminated. By working on the mathematical and statistical concepts involved in this pandemic situation, the teacher can institute new ways of promoting learning, interaction and sharing of meanings among students. Thus, this paper aims to reflect on how teachers can work mathematical and statistical concepts with Elementary School students in the context of the pandemic. We anchored, as technical support, in the Knowledge Base proposed by Shulman, focusing on the specific knowledge of Statistics, pedagogical knowledge and curricular knowledge. We present six Teaching Cases constructed from the data and statistical information available on official websites, aiming to give sense and meaning to the information. For this purpose, we follow the premises of Statistical Literacy, the levels of reading and interpretation of graphs and critical sense. Through these cases, we presented to the teacher different resources, such as concrete materials, simulation through Geogebra applets, indicators, such as, lethality, mortality rate and prevalence from the context of the Covid-19 pandemic. In this perspective, we emphasize the importance of knowing the context and also the development of the capacity of formulating critical questions, in order to contribute to the teacher training, so they can help the students understand the COVID-19 pandemic and take their own decisions. Decisions that will preserve their lives and the lives of the people of their community.
\end{abstract}

Keywords: Statistical Education,. Teacher Training. Knowledge Base. Teaching Case. Covid-19.

\section{Introdução}

A crise sanitária vivenciada pela humanidade evidencia a importância da ciência, em especial da Matemática e da Estatística, na compreensão da Pandemia da Covid-19. Mais do que apenas a compreensão de números e padrões, este fenômeno traz em seu bojo a necessidade de romper os muros da disciplinaridade e a necessidade da transversalidade.
Desta forma, precisamos entender conceitos ligados à Biologia, Ciências, Sociologia, Filosofia e, em especial, ao contexto; desenvolver a capacidade de formular questões críticas, superar nossas crenças e atitudes; e nos posicionar, cada vez mais, em favor de um ensino de Matemática e Estatística que instrumentalize os professores. Somente assim os professores estarão preparados para trabalhar estes

1 Escrito a partir da mesa de discussão virtual de abril/2020, disponível em: https://bit.ly/2WVfVDj . Os dados sobre a Pandemia apresentados neste artigo foram atualizados após a realização da mesa. 
conceitos com seus alunos, a fim de que eles sejam capazes de compreender o mundo e tomar decisões conscientes que vão impactar em sua vida pessoal e em sua comunidade, privilegiando a empatia e a solidariedade na construção de uma sociedade mais justa.

Nas discussões virtuais dos pesquisadores do Grupo de Trabalho Educação Estatística - GT12, da Sociedade Brasileira de Educação Matemática - SBEM, surgiu o seguinte questionamento: a população brasileira compreende as informações estatísticas sobre a Covid-19 divulgadas pela mídia?

Este questionamento nos levou a refletir sobre o nosso papel e compromisso enquanto educadores estatísticos, na formação de uma comunidade letrada estatisticamente. Além disso, a diversidade de informações estatísticas relativas à pandemia provocada pelo novo coronavírus (SARS-CoV-2), causador da doença conhecida como Covid-19, desafiounos a pensar como podemos contribuir para a compreensão das informações estatísticas divulgadas nas mídias. Mais do que isso, como podemos aproveitar essa diversidade de informações matemáticas, estatísticas e de contexto para que o professor de Matemática as possa utilizar em sala de aula?

Como professores e pesquisadores da Educação Estatística, lançamo-nos ao desafio de evidenciar as potencialidades geradas pelos dados da pandemia na formação de professores. A partir deste entendimento surgiu a proposta de uma mesa de discussão virtual que abordou as informações estatísticas da pandemia da Covid-19 apresentadas nos meios de comunicação. Nesta mesa destacamos como os professores poderiam abordar conceitos matemáticos e estatísticos no contexto da pandemia, em especial no que diz respeito à construção e interpretação de gráficos. Participaram desta mesa virtual professores da Educação Básica e do Ensino Superior, estudantes de cursos de Licenciatura e de Programas de Pós-graduação, como o Programa de Educação em Ciências e Matemática. A proposta da mesa se justifica pelos resultados de outras pesquisas na área da Educação Estatística, as quais apontam a necessidade de mais estudos acerca da formação de professores na Educação Estatística (Arteaga, 2011), sobre o desenvolvimento e conhecimento profissional dos professores (Lopes, 2008) e, principalmente, sobre o trabalho com gráficos (Monteiro \& Selva, 2001). O planejamento e organização desta mesa culminou neste artigo.

Como referencial teórico nos reportamos a Shulman (1986, 1987), que postula a Base do Conhecimento, sobretudo o conhecimento específico do conteúdo, que neste caso é formado pelos conceitos de Estatística pontuados pela Base Nacional Comum Curricular - BNCC (Brasil, 2018); o conhecimento pedagógico do conteúdo, que se refere às estratégias que devemos lançar mão para o ensino desse conteúdo; e o conhecimento curricular, também requeridos pela Diretrizes Curriculares Nacionais para a Formação Inicial de Professores para a Educação Básica Resolução Nº 2, de 20 de dezembro de 2019 (Brasil, 2019).

Para Shulman (1986, 1987), o conhecimento específico do conteúdo abarca tanto o conhecimento técnico quanto o de contexto. Assim, para que o professor possa planejar e desenvolver suas aulas não basta o conhecimento específico do conteúdo de Estatística; também se faz necessário compreender como os dados foram gerados, tratados, comunicados e, principalmente, seu contexto, pois este, como pontua Lopes (2013), fornece o significado. Na compreensão dos conceitos estatísticos precisamos do conhecimento matemático, como plano cartesiano e crescimento exponencial; do conhecimento do contexto, como vírus, pandemia, letalidade, taxa de incidência e mortalidade. Sendo assim, recorremos ao Letramento Estatístico, postulado por Gal (2002); aos níveis de compreensão dos gráficos, propostos por Curcio (1989); e senso crítico, conforme Monteiro (2006).

Um sujeito letrado estatisticamente, que compreende os conceitos estatísticos, tem condições de analisar criticamente e argumentar sobre a qualidade e fidedignidade dos dados. Além disso, para a tomada de decisão dos gestores da política pública no controle da pandemia, faz-se necessária a transparência das informações. Esta, por sua vez, é fundamental para evitar a manipulação tendenciosa dos dados, a fim de minimizar ou potencializar os discursos, tendo em vista que a divulgação das informações estatísticas responde aos interesses dos donos dos dados, como ressaltam Cazorla e Castro (2008).

Neste sentido, é preciso formar um professor que seja capaz de ensinar os conteúdos específicos de Matemática/ Estatística, de forma a promover o desenvolvimento de competências e habilidades que possibilitem aos estudantes questionar, argumentar, posicionar-se e tomar decisões para o seu bem e o de sua comunidade, fomentando a formação de cidadãos mais solidários, mais humanos, como preconizado na Base Nacional Comum Curricular (BNCC).

Com isto, propomos que os professores utilizem em suas aulas as informações disponíveis em gráficos, tabelas e medidas resumo, bem como os modelos matemáticos/ estatísticos gerados a partir dos dados da pandemia da Covid-19. Todavia, verificamos que é necessária a busca de informações mais detalhadas, uma vez que essas informações nem sempre explicitam a forma como foram construídas como, por exemplo, o uso de frequência acumulada ou da escala logarítmica, o que pode dificultar a sua compreensão, como alertado em outras situações por Diniz e Borba (2012).

Neste sentido, este artigo tem por objetivo refletir sobre como os professores podem trabalhar conceitos matemáticos e estatísticos com os estudantes da Educação Básica no contexto da pandemia. Para tal, o texto está formado por seis seções. Iniciamos com esta introdução, em que explanamos a motivação e os objetivos do artigo. Na segunda seção, ancorados em Shulman, apresentamos o aporte teórico que fundamenta a discussão sobre a formação de professores; na terceira apresentamos o ensino de Estatística na Educação 
Básica, o Letramento Estatístico, os níveis de compreensão de gráficos e o senso crítico; na quarta expomos o percurso metodológico; na quinta apresentamos seis casos de ensino; e, na sexta seção, tecemos nossas considerações finais.

\section{Aporte Teórico: Formação de Professores}

Defendemos que os cursos de formação de professores e a Educação Básica necessitam superar a organização tradicional de ensino, com uma estrutura curricular fragmentada, na direção de uma organização interdisciplinar, de forma a atender às demandas atuais da sociedade.

Segundo Oliveira (2018, p. 27), “A interdisciplinaridade busca a interação entre as áreas do conhecimento, por meio de planejamentos comuns entre as áreas envolvidas, almejando como resultado um conhecimento menos fragmentado, mais integrado e holístico". Além disso, comungamos com as ideias de Castells (2003, p. 227), pois "precisamos de uma nova pedagogia, baseada na interatividade, na personalização e no desenvolvimento da capacidade autônoma de aprender e pensar". Para esse autor, as transformações que a sociedade vem passando ao longo do tempo apontam para a necessidade de formar indivíduos capazes de obter a informação, recombinando-a e usando-a para produzir conhecimento. Para isso, segundo Torre (2004), os processos educativos necessitam investir no desenvolvimento profissional docente em termos de conhecimentos, atitudes, hábitos, crenças e habilidades, tanto pessoais como sociais.

O ensino de Estatística, pela sua própria natureza, requer uma interlocução que vai além do conhecimento específico, dos conceitos e procedimentos matemáticos subjacentes, haja vista que o conhecimento do contexto tem papel fundamental. Por essa razão, recorremos ao aporte teórico postulado por Lee Shulman que, na década de 1980, propôs uma Base do Conhecimento profissional para o ensino que tem sido referência em diversos países. A Base proposta por Shulman (1986, 1987) deriva, em boa parte, da observação e análise da prática de professores no que concerne, em especial, ao conteúdo a ser ensinado e sua relação com as estratégias pedagógicas empregadas pelos docentes.

Por meio de entrevistas, observações, atividades e exame de materiais, temos tentado entender como eles trafegam, na ida e na volta, do status de aprendiz para o de professor, de ser capaz de compreender o conteúdo por si mesmo a se tornar capaz de elucidar o conteúdo de novas maneiras, reorganizálo e dividi-lo, envolvê-lo em atividades e emoções, em metáforas e exercícios, e em exemplos e demonstrações, de forma que possa ser compreendido pelos alunos. (Shulman, 2014, p. 213).

Ao investigar o conhecimento que os professores têm dos conteúdos de ensino, as relações que estabelecem entre esses e a forma como os organizam e apresentam aos estudantes, Shulman pôde compreender o que os professores sabiam (ou não) que lhes permitia ensinar de uma certa maneira. Para o autor, os professores são sujeitos que possuem uma história de vida pessoal e profissional e produzem e mobilizam saberes no exercício de sua prática.

Assim, a Base de Conhecimento proposta por Shulman (1986) consiste de um corpo teórico de compreensões, atitudes e métodos que um professor mobiliza para transformar o conhecimento que possui do conteúdo em formas de atuação que sejam pedagogicamente efetivas e adaptáveis às diversas habilidades e repertórios dos alunos.

A partir deste estudo, Shulman (1986) estabeleceu categorias de ensino: conhecimento do conteúdo específico; conhecimento pedagógico do conteúdo e conhecimento curricular do conteúdo. $\mathrm{O}$ conhecimento do conteúdo específico se refere ao entendimento do professor em relação aos conceitos e à compreensão dos processos de sua produção. O conhecimento pedagógico do conteúdo vai além do conhecimento do conteúdo a ser ensinado. Esta categoria envolve a capacidade do professor em transformar o conhecimento que ele possuiu em representações que tornem os conceitos compreensíveis aos estudantes. O professor pode utilizar diversas formas de representar os conceitos, como animações, demonstrações e explicações, as quais ele vai adquirindo ao longo de sua prática docente ou por meio de pesquisas. Esta variedade de representações propicia tanto atender as diferentes formas de aprender dos estudantes como superar as dificuldades que estes encontram na compreensão dos conceitos. O conhecimento curricular, por sua vez envolve, o conhecimento do professor sobre as alternativas curriculares disponíveis para o ensino e compreende duas modalidades: horizontal e vertical. A modalidade horizontal diz respeito ao professor estar familiarizado com os conteúdos curriculares estudados em outras disciplinas (interdisciplinaridade) no mesmo ano ou semestre letivo. A modalidade vertical equivale a familiaridade do professor tanto com os conteúdos ou questões que foram discutidas no semestre ou ano escolar anterior, quanto com os que serão discutidos nas próximas disciplinas (Shulman, 1986).

Neste momento, cabe ressaltar que, na maioria das vezes, essas três categorias da Base do Conhecimento propostas por Shulman (1986) se sobrepõem. Tal imbricamento é destacado por Miola e Pereira (2012) em estudo com um grupo de professores de Matemática. Para as autoras, a prática docente exige um amplo conhecimento do professor sobre sua disciplina e dos conteúdos a serem ensinados, de modo que a deficiência em relação a esses conhecimentos (do conteúdo, pedagógico e curricular) pode prejudicar a escolha do material didático e do planejamento da aula comprometendo, consequentemente, a aprendizagem dos estudantes.

Tal situação no campo da Estatística é evidenciada por Arteaga (2011), que apresenta os fundamentos da formação de professores para ensinar Estatística, dentre os quais destacamos os conhecimentos de diferentes naturezas associados a um conteúdo e o uso de diferentes abordagens metodológicas em níveis de ensino, com bons exemplos de atividades, a partir da análise crítica dos livros e materiais didáticos, buscando prever as dificuldades e refletir sobre os erros que os alunos 
possuem/realizam na resolução de problemas.

Além disto, estudos desenvolvidos por Arteaga (2011) e Monteiro e Selva (2001) apontam que os professores relatam dificuldades na compreensão de conceitos da Estatística que precisam ensinar a seus alunos e, portanto, afirmam estarem despreparados para tal, o que torna o ensino de Estatística um desafio para os docentes.

Dizer que um professor deve antes compreender tanto o conteúdo como os propósitos, no entanto, não distingue especificamente o professor dos não professores. Espera-se que um matemático entenda matemática e que um historiador compreenda história. Mas a chave para distinguir a base de conhecimento para o ensino está na interseção entre conteúdo e pedagogia, na capacidade do professor para transformar o conhecimento de conteúdo que possui em formas que são pedagogicamente poderosas e, mesmo assim, adaptáveis às variações em habilidade e histórico apresentadas pelos alunos. (Shulman, 2014, p. 217).

As novas compreensões dos propósitos educacionais, dos materiais adotados, do trabalho interdisciplinar, da compreensão das formas de aprender dos alunos e de si mesmo não ocorrem automaticamente. Para isso, é preciso encontrar uma ponte entre a compreensão do professor e aquela que ele deseja para os alunos, o que envolve selecionar materiais didáticos, organizar a sala de aula, apresentar explicações claras, atribuir trabalhos que possibilitem interagir com os estudantes e promover a discussão e reflexão crítica. Assim, segundo Shulman (2014), não basta o professor ter o conhecimento do conteúdo; ele precisa também ter o conhecimento pedagógico do conteúdo. Além disso, o conhecimento curricular é fundamental neste processo para que o professor possa articular o ensino de Estatística dentro da própria Matemática e com as diversas áreas de conhecimentos de onde surgem os dados que, neste caso, são da pandemia da Covid-19. A partir do contexto da pandemia é possível articular conceitos de Matemática e Estatística com outros advindos da área de Ciências Humanas e Sociais, Ciências Naturais, Linguagens e temas transversais como saúde, economia, meio ambiente, trabalho, entre outros, pontuados na BNCC.

A partir do exposto, assumimos que ao ensinar os conteúdos específicos de Estatística e Matemática precisamos dialogar com as outras áreas de conhecimento, sendo que os Casos de Ensino aqui propostos têm essa intencionalidade. Para Shulman (1986), um Caso de Ensino tem como proposta apresentar uma situação que possa ser discutida no coletivo, relacionada a outros saberes e refletida com base em compreensões teóricas. Conforme o autor, um Caso de Ensino deve ser debatido, explicado, interpretado, analisado e remontado. Isso exige do professor tanto o conhecimento do conteúdo específico que pretende ensinar como estratégias pedagógicas que podem ser adotadas para que os estudantes aprendam.

Dois casos de ensino envolvendo a média aritmética e suas propriedades foram apresentados por Silva, Alves, Pietropaolo e Amorim (2020), no qual discutem os resultados da interação dos professores. Já neste trabalho propomos seis casos de ensino explorando alguns aspectos das informações geradas pela Covid-19, deixando em aberto outras possibilidades.

Com este entendimento, este artigo apresenta reflexões sobre a potencialidade do uso das informações geradas pela pandemia da Covid-19, o que pode auxiliar o professor de Matemática no seu desenvolvimento profissional, elucidando o conteúdo estatístico e novas maneiras de reorganizá-los e envolvê-los em exemplos, demonstrações e atividades neste contexto, de forma que tanto os conceitos estatísticos quanto a situação pandêmica possam ser compreendidos.

\section{O Desafio de Ensinar Estatística na Educação Básica}

Para Cobb e Moore (1997), a Estatística auxilia outros campos de conhecimento, fornecendo métodos e técnicas para analisar dados. Por sua vez, a Educação Estatística é um campo interdisciplinar que foca o ensino e a aprendizagem de Estatística (Zieffler, Garfield \& Fry, 2018). Na perspectiva de Garfield e Ben-Zvi (2008), a Educação Estatística se ancora na Educação Matemática, que fornece arcabouços teóricos metodológicos da pesquisa relativa à aprendizagem e desenvolvimento da cognição, mas que evoluiu como um campo independente de investigação.

A preocupação com o ensino e a aprendizagem de Estatística se intensificou quando os países inseriram seus conteúdos nos currículos da Educação Básica, passando de uma visão centrada em treinamentos, focada nos procedimentos e ferramentas matemáticas, para uma educação de pessoas capazes de utilizar o conhecimento estatístico na leitura de mundo, denominado de letramento estatístico.

No Brasil, a inserção dos conteúdos de Estatística na Educação Básica foi oficializada com a publicação dos Parâmetros Curriculares Nacionais (PCN) na disciplina de Matemática, de modo que este documento já destacava a importância da Estatística na tomada de decisões diante de questões políticas e sociais. Essa inserção foi ratificada pela Base Nacional Comum Curricular (BNCC) como uma unidade temática de Matemática, denominada "Probabilidade e Estatística" (Brasil, 2018). A BNCC elenca dez competências gerais que devem ser desenvolvidas durante o Ensino Fundamental, dentre as quais destacamos duas:

Competência 7: Argumentar com base em fatos, dados e informações confiáveis, para formular, negociar e defender ideias, pontos de vista e decisões comuns que respeitem e promovam os direitos humanos, a consciência socioambiental e o consumo responsável em âmbito local, regional e global, com posicionamento ético em relação ao cuidado de si mesmo, dos outros e do planeta. .

Competência 10: Agir pessoal e coletivamente com autonomia, responsabilidade, flexibilidade, resiliência e determinação, tomando decisões com base em princípios éticos, democráticos, inclusivos, sustentáveis e solidários (Brasil, 2018, p. 9 - grifo dos autores).

Relativamente às competências que devem ser desenvolvidas no ensino da Matemática, salientamos a quarta 
e a sétima:

Fazer observações sistemáticas de aspectos quantitativos e qualitativos presentes nas práticas sociais e culturais, de modo a investigar, organizar, representar e comunicar informações relevantes, para interpretá-las e avaliá-las crítica e eticamente, produzindo argumentos convincentes ...] Desenvolver e/ou discutir projetos que abordem, sobretudo, questões de urgência social, com base em princípios éticos, democráticos, sustentáveis e solidários, valorizando a diversidade de opiniões de indivíduos e de grupos sociais, sem preconceitos de qualquer natureza. (Brasil, 2018, p. 267).

Para atender às demandas da BNCC apresentamos nossa proposta de Casos de Ensino ancorada no letramento estatístico postulado por Gal (2002), nos níveis de compreensão de gráficos, de Curcio (1989) e no senso crítico, de Monteiro (2006).

Para Gal (2002), o letramento estatístico se refere, em termos gerais, a dois componentes inter-relacionados: i) a capacidade de interpretar e avaliar criticamente as informações estatísticas, os argumentos relacionados aos dados ou aos fenômenos estocásticos, os quais podem ser encontrados em diversos contextos; e, quando relevante, ii) a capacidade de discutir ou comunicar suas reações a tais informações estatísticas, como a compreensão do significado da informação, opiniões sobre as implicações das informações ou as preocupações quanto à aceitabilidade de determinadas conclusões.

O modelo proposto pelo autor envolve dois componentes: o cognitivo e o atitudinal. O componente cognitivo é formado por cinco elementos que são responsáveis pela competência das pessoas para compreender, interpretar e avaliar criticamente as informações estatísticas, são eles: letramento; conhecimento estatístico; conhecimento matemático; conhecimento do contexto; e a competência para elaborar questões. O componente atitudinal, por sua vez, é formado por dois elementos. $\mathrm{O}$ primeiro relacionado às atitudes e às crenças das pessoas, que moldam suas visões de mundo; e o segundo relacionado à postura crítica, que nada mais é que a aptidão para uma conduta questionadora diante das informações estatísticas.

Especificamente para a compreensão de informações estatísticas contidas em gráficos, recorremos aos níveis de leitura propostos por Curcio (1989), a saber:

- Nível 1 - "Ler os dados": leitura pontual dos gráficos, não necessitando que o leitor saiba interpretar os dados para além do que está efetivamente posto e perceptível na representação, tendo um custo cognitivo muito baixo. Por exemplo, encontrar o valor para um ano de uma variável no gráfico;

- Nível 2 - "Ler entre os dados": inclui a interpretação e integração dos dados no gráfico e requer a habilidade de comparar quantidades e o uso de outros conceitos matemáticos. Esse tipo de leitura requer, ao menos, um grau de inferência lógica ou pragmática como, por exemplo, identificar em que período houve maior crescimento ou a variável que sofreu maior variação ou seu valor máximo;

- Nível 3 - "Ler além dos dados": esse é um nível mais complexo e solicita do leitor uma interpretação global, não apenas dos dados contidos no gráfico, mas da inferência ou predição de um acontecimento ou resultado.

Além disso, precisamos de uma leitura crítica das informações contidas nos gráficos e, para isso, recorremos a Monteiro (2006), que denomina de senso crítico o processo de compreensão dos gráficos e sinaliza que, além dos conhecimentos matemáticos/estatísticos, podemos mobilizar expressões afetivas (sentimentos e emoções), exemplificação pessoal e referência contextual (conhecimentos do tipo social, político e/ou econômico) relacionados ao tema do gráfico.

Utilizamos o senso crítico para sinalizar aos professores como podemos iniciar a compreensão das informações estatísticas da Covid-19 e, assim, desenvolver interpretações mais acuradas, propiciando condições para a capacidade de elaborar questões críticas, aguçando a curiosidade e a busca de compreensão da pandemia da Covid-19 na Educação Básica.

\section{Percurso Metodológico}

Acompanhando as informações divulgadas na mídia sobre a Pandemia da Covid-19, percebemos a relevância da Estatística na sua compreensão por parte da população, no auxílio da tomada de decisão e no planejamento de estratégias por parte dos gestores públicos a fim de conter a disseminação desta doença. Também, percebemos a falta do letramento estatístico de muitos jornalistas, bem como interpretações equivocadas no que diz respeito às taxas, tabelas e gráficos veiculados na mídia.

Neste contexto, este artigo apresenta seis Casos de Ensino envolvendo o contexto da pandemia da Covid-19, a fim de refletir como os professores podem trabalhar em suas aulas alguns conhecimentos de Estatística, Matemática e Epidemiologia. Para tal, traçamos abordagens pedagógicas e abordamos a pertinência desses conceitos de acordo com o currículo escolar.

Assim, discutimos a importância de compreender e distinguir os números absolutos dos relativos e, dentre os relativos, a importância do valor que vai no denominador, como nos conceitos de letalidade, taxa de incidência e mortalidade; acompanhamos a evolução da pandemia em gráficos, focando os níveis de compreensão de Curcio (1989) e o senso crítico de Monteiro (2006); e, por fim, exploramos ideias de experimentação com a função exponencial para modelar teoricamente a evolução do número de casos, o achatamento da curva, disponibilizando dois applets no Geogebra.

\section{Casos de Ensino}

Para Shulman (1986, 1987), a prática pedagógica e os métodos de ensino ativos e interativos só serão adotados pelo professor quando este domina e compreende os conteúdos a serem ensinados. Neste sentido, apresentamos seis Casos de Ensino que possibilitam ao professor trabalhar os conceitos Matemáticos e Estatísticos por meio de uma abordagem científica alinhada com as principais pesquisas sobre o 
assunto. Estas pesquisas têm evidenciado a relevância do isolamento social para diminuir o número de casos e de óbitos, "achatando a curva" para que o sistema hospitalar possa atender os doentes de forma digna.

Antes de iniciar os Casos de Ensino, pontuamos dois cenários. No primeiro, o professor de Matemática pode fazer uma interlocução com colegas de outras disciplinas, montando uma estratégia genuinamente interdisciplinar; no segundo, caso o professor não consiga essa articulação, ainda pode trabalhar desde uma perspectiva interdisciplinar, porém, buscando subsídios sobre os conceitos associados às outras áreas do conhecimento.

Os primeiros conceitos a serem trabalhados pelo professor devem estar ligados à própria diferença entre as palavras epidemia e pandemia. Epidemia ocorre quando há um aumento do número de casos de determinada doença, muito acima do esperado e não delimitado apenas a uma região; já pandemia, por sua vez, ocorre quando o número de casos da doença fica acima do esperado, afetando vários países e continentes (Brasil, 2002). Esses conceitos podem ser trabalhados fazendo referências a outras pandemias, tais como a varíola, gripe espanhola, Síndrome Respiratória Aguda Grave (SARS), Síndrome Respiratória do Oriente Médio (MERS) e a Síndrome da Imunodeficiência Adquirida (AIDS), causada pelo Vírus da Imunodeficiência Humana (HIV), articulandoos às diversas áreas do conhecimento.

No caso específico da pandemia da Covid-19, a sua rápida disseminação se deve à mobilidade internacional, o que torna a humanidade ainda mais vulnerável à ocorrência de novas crises sanitárias. Além disso, aproximadamente 80\% dos infectados não desenvolvem os sintomas (assintomáticos), mas disseminam a doença. As peculiaridades desta pandemia são extremamente importantes para interpretação e comparação de dados, pois ajudam a entender contextos históricos e geográficos de onde os dados se originam.

Com esse pano de fundo, o professor pode propor diversas reflexões acerca de diferentes conteúdos para o ensino de Matemática e Estatística, como por exemplo: conjuntos numéricos e as suas operações, interpretação de gráficos, porcentagem, probabilidade, progressão geométrica, função exponencial, função logarítmica, entre outros, o que implica no conhecimento do currículo de Matemática/Estatística e das outras disciplinas para elencar os conhecimentos prévios necessários para trabalhar os conceitos envolvidos na pandemia no Ensino Fundamental ou Médio. Dessa forma, iniciamos a reflexão com os professores trazendo à tona a relevância do papel social que a Matemática pode exercer quando utilizamos as informações oficiais para propor estratégias de ensino.

\subsection{Caso de Ensino 1: Números absolutos e relativos}

O professor pode iniciar a partir dos painéis da Covid-19 em nível mundial, do Brasil, do estado ou do município onde está inserida a escola. Esses painéis trazem um panorama geral da Covid-19 envolvendo duas variáveis estatísticas chave: "Número de casos confirmados $(\mathrm{k})^{2}$ ", definido como o número de pessoas doentes com teste positivo, e "Número de mortes $(\mathrm{m})^{3}$ ", definido como os casos confirmados que vieram a óbito. Das pessoas que fazem parte dessas estatísticas foram coletadas algumas informações que permitem traçar o perfil epidemiológico dos que sofreram a doença e dos que morreram, tais como: sexo, idade, raça e comorbidades (doenças préexistentes, obesidade etc.). Diversos países seguem regras internacionais de notificação das doenças. No caso do Brasil, o Sistema de Vigilância Epidemiológica, implementado em cada município, informa para o seu estado, que repassa a informação para o Ministério da Saúde, que por sua vez alimenta o painel mundial da Covid-19, coordenado pela Organização Mundial da Saúde - OMS, que decretou o estado de pandemia.

O professor também pode explorar outros conceitos, como a taxa de incidência e de mortalidade. A taxa de incidência é calculada como a razão entre o número de casos confirmados da doença $(\mathrm{k})$ e o total da população $(\mathrm{N})$ e expressa a velocidade com que a doença vai se espalhando pela população. A taxa de mortalidade é calculada como a razão entre número de mortes (m) e o total da população $(\mathrm{N})$, sendo interpretada como a parcela relativa da população que morreu dessa doença. É importante observar que essas duas taxas têm o mesmo denominador que, em geral, é o total da população de um país, estado, município ou região. Como estas duas taxas possuem valores muito pequenos, normalmente são apresentadas por milhão ou por cem mil habitantes. Já a letalidade é apresentada em porcentagem e consiste na proporção entre o número de mortes $(\mathrm{m})$ e número de casos confirmados. Podemos interpretar esta medida como o risco de morrer entre os doentes. Na Figura 1 exemplificamos estas taxas.

Figura 1 - Conceitos epidemiológicos

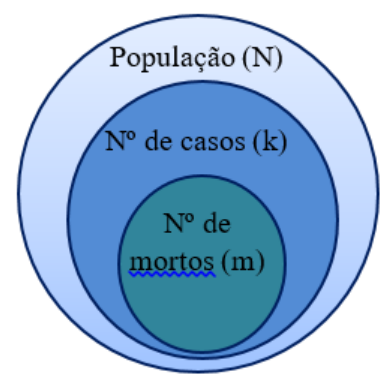

TaxaIncidência $=$ 1.000.000* (Número de casos) $/$ População $=$ $1.000 .000 * \mathrm{k} / \mathrm{N}$

TaxaMortalidade $=$ 1.000.000* (Número de mortos) / População = $1.000 .000 * \mathrm{~m} / \mathrm{N}$

Letalidade $=100^{*}$ (Número de Mortos) / (Número de casos) $=$ $100 * \mathrm{~m} / \mathrm{k}$

Fonte: Construção dos autores.

2 A maioria dos países considera a variável "número de casos" como o número de pessoas que realizaram o teste para novo coronavírus (SARS-CoV-2) com resultado positivo, denominados também de casos confirmados.

$3 \mathrm{O}$ número de mortes tem uma maior variação na definição. Alguns países só contabilizam os óbitos ocorridos nos hospitais mediante confirmação do teste, enquanto outros países contabilizam os casos suspeitos, ou seja, sem o resultado do teste, além dos hospitais, em casa e em casas de repouso. 


\subsection{Caso de Ensino 2: Representações gráficas}

Para exemplificar estas medidas e compreender a pandemia em nível mundial, os professores podem utilizar o Infográfico da Covid-19, criado pela Universidade de Johns Hopkins (Figura 2), que é formado por cinco gráficos. $\mathrm{O}$ primeiro apresenta o número de casos confirmados $(\mathrm{k})$ e o segundo o número de mortes $(\mathrm{m})$, ambos em gráfico de barras; e o terceiro mostra a letalidade em porcentagem $(\mathrm{m} / \mathrm{k})$, sendo utilizado o gráfico de bastão. Estes três gráficos estão ordenados pelo número de casos confirmados. O quarto gráfico, de barras, apresenta a taxa de incidência e o quinto gráfico, de pontos, apresenta a taxa de mortalidade, estes dois últimos, ordenados de forma independente.

O professor pode iniciar a discussão perguntando: qual é a situação do Brasil em 13 de maio de 2020? Neste caso, a leitura requer apenas a extração dos dados, verificando que o país ocupava a $7^{\mathrm{a}}$ posição do ranking em número de casos, a $6^{\mathrm{a}}$ posição no número de mortes, o $18^{\circ}$ lugar no ranking de mortalidade, mas não aparece no ranking da taxa de incidência, o que requer uma leitura de nível 1, ler os dados, segundo Curcio (1989). Aqui o professor pode perguntar: Por que o Brasil não aparece nesse ranking? Para os estudantes responderem a esta pergunta, precisam relacionar as informações com o tamanho da população dos países, o que exige uma leitura de nível 3, para além dos dados (Curcio, 1989) e referência contextual de Monteiro (2006). Atualmente, a população do Brasil é de aproximadamente 210 milhões de habitantes, enquanto que a maioria dos países europeus não supera 80 milhões. Esta é a explicação, já que este valor está no denominador dessa taxa.

Figura 2 - Número de casos e taxas da Covid-19 nos países mais atingidos

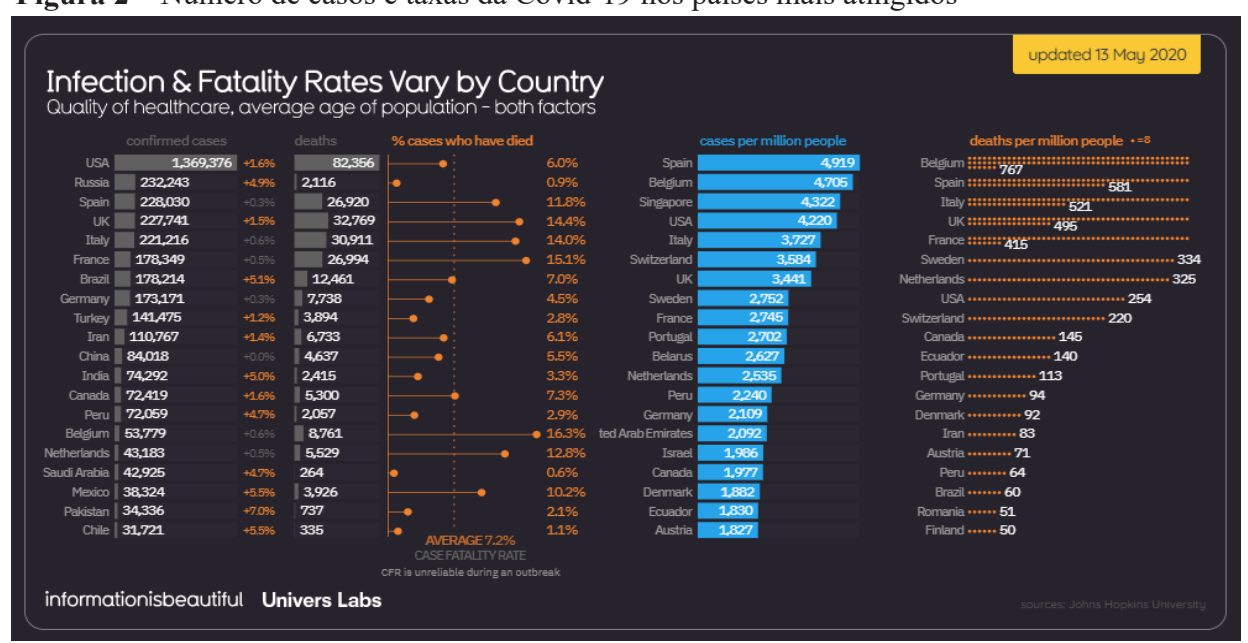

Fonte: https://informationisbeautiful.net/visualizations/covid-19-coronavirus-infographic-datapack/

Caso o professor tenha interesse em obter informações sobre a taxa de incidência e de mortalidade, ele pode recorrer ao Painel do Worldometers (Figura 3), que se baseia em dados da OMS. Além das informações da Figura 2, este também apresenta o número de casos críticos e número de testes realizados pelos países.

Figura 3 - Número de casos por tipo, mortes, taxa de incidência e mortalidade e número de testes da Covid-19 nos países mais atingidos em 13 de maio de 2020

\begin{tabular}{|c|c|c|c|c|c|c|c|c|c|c|c|}
\hline Europe & North At & nerica & Sout & America & Africa & ania & & & & & \\
\hline $\begin{array}{l}\text { Country, } \\
\text { Other }\end{array}$ & $\begin{array}{l}\text { Total } \\
\text { Cases } \mid \equiv\end{array}$ & $\begin{array}{l}\text { New } \\
\text { Cases }\end{array}$ & $\begin{array}{l}\text { Total } \\
\text { Deaths }\end{array}$ & $\begin{array}{l}\text { New } \\
\text { Deaths }\end{array}$ & $\begin{array}{l}\text { Total } \\
\text { Recovered }\end{array}$ & $\begin{array}{l}\text { Active } \\
\text { Cases }\end{array}$ & $\begin{array}{l}\text { Serious, } \\
\text { Critical t1 }\end{array}$ & $\begin{array}{l}\text { Tot Cases/ } \\
\text { 1M pop }\end{array}$ & $\begin{array}{l}\text { Deaths/ } \\
\text { 1M pop }\end{array}$ & $\begin{array}{l}\text { Total } \\
\text { Tests }\end{array}$ & $\begin{array}{l}\text { Tests/ } \\
\text { 1M pop }\end{array}$ \\
\hline World & $4,399,090$ & $+61,653$ & 296,092 & $+3,641$ & $1,644,702$ & $2,458,296$ & 45,899 & 564 & 38.0 & & \\
\hline$\underline{\text { USA }}$ & $1,420,421$ & $+11,785$ & 84,353 & +928 & 305,723 & $1,030,345$ & 16,373 & 4,291 & 255 & $10,061,909$ & 30,398 \\
\hline Spain & 271,095 & $+1,575$ & 27,104 & +184 & 183,227 & 60,764 & 1,534 & 5,798 & 580 & $2,467,761$ & 52,781 \\
\hline Russia & 242,271 & $+10,028$ & 2,212 & +96 & 48,003 & 192,056 & 2,300 & 1,660 & 15 & $5,982,558$ & 40,995 \\
\hline$\underline{\text { UK }}$ & 229,705 & $+3,242$ & 33,186 & +494 & N/A & 196,175 & 1,559 & 3,384 & 489 & $2,094,209$ & 30,849 \\
\hline Italy. & 222,104 & +888 & 31,106 & +195 & 112,541 & 78,457 & 893 & 3,673 & 514 & $2,735,628$ & 45,246 \\
\hline Brazil & 180,737 & $+3,135$ & 12,635 & +231 & 72,597 & 95,505 & 8,318 & 850 & 59 & 735,224 & 3,459 \\
\hline France & 178,060 & & 27,074 & +83 & 58,673 & 92,313 & 2,428 & 2,728 & 415 & $1,384,633$ & 21,213 \\
\hline Germany. & 173,824 & +653 & 7,792 & +54 & 148,700 & 17,332 & 1,465 & 2,075 & 93 & $3,147,771$ & 37,570 \\
\hline Turkey. & 143,114 & $+1,639$ & 3,952 & +58 & 101,715 & 37,447 & 998 & 1,697 & 47 & $1,474,003$ & 17,477 \\
\hline Iran & 112,725 & $+1,958$ & 6,783 & +50 & 89,428 & 16,514 & 2,735 & 1,342 & 81 & 629,534 & 7,495 \\
\hline
\end{tabular}

Fonte: https://www.worldometers.info/coronavirus/\#countries. 
Para leitura dos dados, nível 2 e 3 de Curcio (1989), o professor pode solicitar que os estudantes indiquem em qual país o problema é mais grave. Os estudantes podem notar que, ao considerarem os números absolutos de casos e mortes, o país classificado em primeiro lugar do ranking é Estados Unidos. No entanto, ao considerar as taxas de incidência e mortalidade, a Espanha e a Bélgica lideram o ranking. $\mathrm{O}$ professor pode indagar: por que isto acontece? A resposta seria a natureza das taxas, que têm como denominador a população de cada país, tornando a comparação mais adequada, pois os países têm populações diferentes. Por esse motivo é que em 13 de maio de 2020 um total de 8.761 casos de mortes na Bélgica reflete em uma taxa de mortalidade de 767 mortes por 1.000.000 de pessoas, enquanto que os Estados Unidos, que apresenta 82.356 mortes na mesma data, tem uma taxa de mortalidade de 254 mortes por milhão. Com isso, é importante esclarecer que, para comparações entre grupos (países, estados, subgrupos da população) que tenham população de tamanhos diferentes, não devemos usar os números absolutos, mas os números relativos, como as taxas mencionadas. Esta situação exige do professor o conhecimento específico do conteúdo a ser ensinado, assim como o conhecimento de contexto, os quais constituem uma das categorias da Base do Conhecimento propostas por Shulman (1986).

Isso não significa que os números absolutos não sirvam, ao contrário, esses são muito úteis para dimensionar a estrutura hospitalar, como número de leitos na Unidade de Terapia Intensiva (UTI), de ambulâncias e de testes da doença, por exemplo. Os dois tipos de números conduzem a resultados importantes sobre o fenômeno investigado, sendo que cada um têm seu papel, seu lugar e sua contribuição, não havendo sentido em atribuir prioridade a um sobre o outro.

Observando, ainda, a Figura 2, quanto à informação referente à letalidade, o professor de Matemática pode perguntar aos estudantes qual interpretação podem fazer sobre essa medida epidemiológica. Nesta, é possível notar que Bélgica, França, Reino Unido, Itália e Holanda apresentam os maiores valores, enquanto os Estados Unidos, que totaliza quase a soma das mortes do Reino Unido, Itália e França, apresenta letalidade com valor próximo à metade de cada um desses países. O professor de Matemática pode perguntar: o que pode influenciar alguns países terem letalidades tão diferentes? Ao observar o cálculo dessa medida, é possível notar que o denominador é o total de casos confirmados da doença, que podemos interpretar como a probabilidade de morrer. Assim, se um país recomenda que as pessoas só procurem os hospitais com sintomas graves e somente estes são testados para Covid-19, isto implica em uma maior letalidade; enquanto que se um país testa também os casos leves, sem a necessidade de hospitalização, a letalidade será menor.

No Brasil, por exemplo, a recomendação para os casos leves da doença foi ficar em casa por não ter capacidade hospitalar para atendimento e nem testes para a população, seja por dificuldade logística, tamanho da população ou por escassez de testes no mercado mundial. Com isto, a taxa de letalidade no Brasil não reflete a probabilidade de morrer entre os doentes, mas a de morrer entre os casos mais graves.

Estes questionamentos estão relacionados ao senso crítico de Monteiro (2006), pois permitem que experiências e conhecimentos prévios sobre a pandemia sejam manifestados pelos estudantes. Estes conhecimentos prévios podem ser de natureza social, cultural e econômica (referência contextual), expressões afetivas (sentimentos e emoções) e exemplificações pessoais, os quais possibilitam criar as condições que permitirão aos estudantes compararem os dados entre os países e, também, entre as variáveis apresentadas.

Ainda nesta seara, se o professor de Matemática quiser aprofundar o contexto, pode solicitar aos estudantes que façam uma investigação dos motivos pelos quais a Bélgica tem uma alta taxa de mortalidade. Este país contabiliza também as mortes nas residências e em casas de repouso, mesmo sem teste, apenas com o diagnóstico clínico. Já a Espanha contabiliza apenas os óbitos ocorridos nos hospitais com teste confirmado. Essa diferente forma de contabilizar os números interfere no ranking, o que exige uma interpretação mais cuidadosa, pois um país com valores baixos da taxa de mortalidade pode significar pouco registro de mortes e não pouca ocorrência. Logo, é preciso ir além da leitura de tendências matemáticas e estatísticas, buscando informações do contexto e interpretando-as criticamente.

\subsection{Caso de Ensino 3: Aplicação da escala logarítmica na representação gráfica}

Ainda na compreensão da evolução da pandemia, ao longo do tempo, verificamos que a evolução do número de casos segue uma curva exponencial, como apresentaremos mais adiante (na Figura 6). Todavia, é de praxe realizar esta análise utilizando a transformação logarítmica, como podemos observar na Figura 4. Este gráfico apresenta informações relevantes para acompanhar a evolução da pandemia. Podemos verificar que nos primeiros 30 dias a maioria das trajetórias dos países era linear, isto é, o crescimento não era exponencial; nos 30 dias seguintes a maioria das trajetórias ainda cresce, mas de forma menos acelerada, sendo que nos últimos 30 dias alguns países se estabilizam, como a China e a Coreia do Sul.

Aqui o professor, em sua aula, pode explorar o significado do logaritmo, neste caso, base 10. Isso significa que quando há casos de 0 a 10 , na escala logarítmica equivale de 0 a 1 ; quando os casos variam de 10 a 100 , na escala logarítmica varia de 1 a 2; quando os casos variam de 101 a 1.000, na escala varia de 2 a 3 e assim sucessivamente. 
Figura 4 - Número de casos da Covid-19 em escala logarítmica

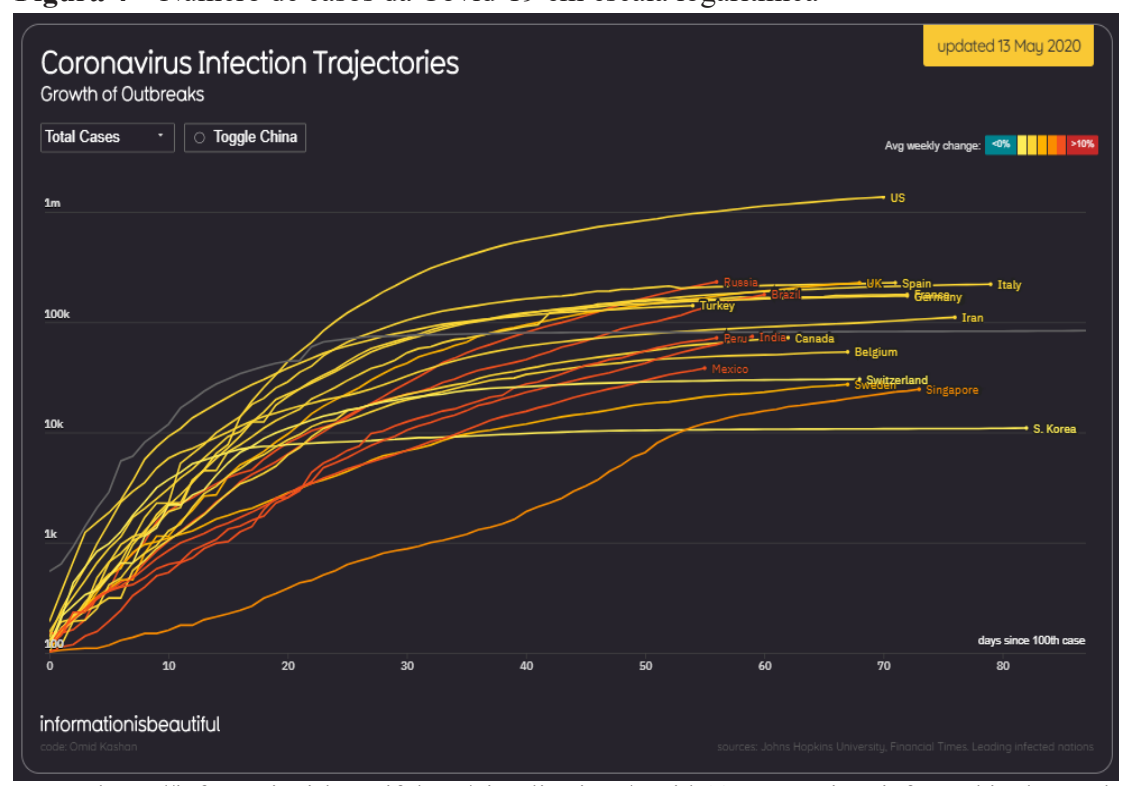

Fonte: https://informationisbeautiful.net/visualizations/covid-19-coronavirus-infographic-datapack/.

Na Figura 4 podemos observar que as trajetórias dos países estão coloridas desde azul, passando por amarelo, laranja e chegando ao vermelho. Quanto mais próxima da cor vermelha maior é a taxa de crescimento. Assim, podemos ver que o Brasil, a Rússia, o México, o Peru e a Índia apresentavam trajetórias ainda crescentes.

Além dos elementos já pontuados por Curcio (1989) e Monteiro (2006), defendemos que, por meio da Educação Matemática Crítica (Skovsmose, 2007) e da Etnomatemática (D'Ambrosio, 2005), as realidades particulares sejam analisadas criticamente, com múltiplos olhares a partir dos cálculos matemáticos e estatísticos, refletindo sobre o uso em diferentes contextos, interpretando esses sinais, códigos e modelos matemáticos e estatísticos para a compreensão da realidade investigada. Segundo Shulman (2014, p.215), o entendimento desta realidade surge "a partir do ponto de vista do professor, que está diante do desafio de tomar o que já compreende e prepará-lo para um ensino eficaz" (p. 215).

\subsection{Caso de Ensino 4: Informações oficiais sobre a Pandemia}

Para analisar os dados do Brasil, podemos utilizar o painel do Ministério da Saúde, que fornece informações sobre o número de casos confirmados e o número de óbitos por estados, dentre outros, como mostramos na Figura 5.

Figura 5 - Painel de casos da Covid-19 no Brasil pelo Ministério da Saúde, em 13 de maio de 2020

\section{CORONAVÍRUS /| BRASIL}

Painel Coronavírus

Atualizado en: 12/05/2020 19:22

\begin{tabular}{|c|c|c|c|}
\hline \multicolumn{2}{|c|}{ CASOS CONFIRMADOS } & & ÓBITOS CONFIRMADOS \\
\hline $\begin{array}{c}177.589 \\
\text { Acumuladolo }\end{array}$ & $\begin{array}{l}9.258 \\
\text { Registros nowas }\end{array}$ & & $\begin{array}{l}881 \\
\text { Regalistros novos }\end{array}$ \\
\hline $\begin{array}{l}92.593 \\
\text { Em soconperathat }\end{array}$ & $\begin{array}{l}72.597 \\
\text { of Recuperados }\end{array}$ & & 5,9 \\
\hline $\begin{array}{l}84,5 \\
\text { Incidenciat }\end{array}$ & & & \\
\hline
\end{tabular}

Fonte: https://covid.saude.gov.br/

Na Figura 6 apresentamos a evolução do número de casos e de mortes no Brasil e podemos verificar o crescimento exponencial. Na Figura 7 apresentamos os mesmos dados na escala logarítmica. 
Figura 6 - Número de casos confirmados e de óbitos do Brasil, em 13 de maio de 2020

Óbitos acumulados de COVID-19 por data de notificação

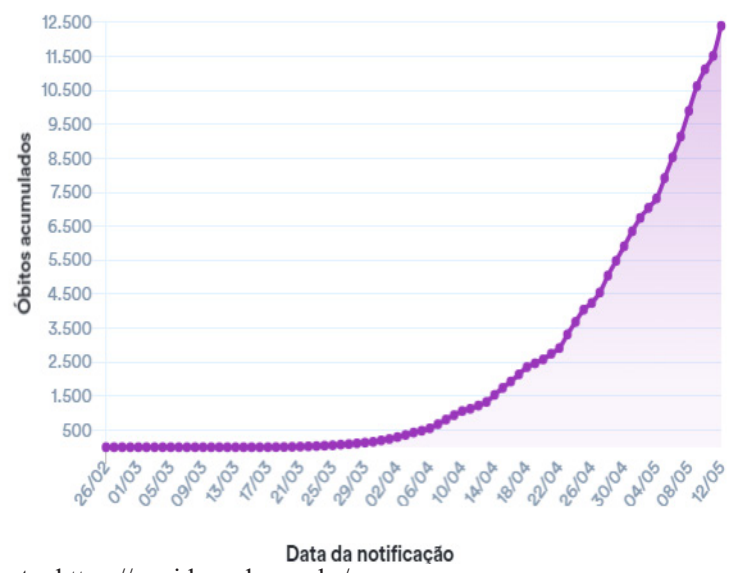

Fonte: https://covid.saude.gov.br/

Figura 7 - Logaritmo, base 10, do número de casos confirmados e de óbitos do Brasil, em 13 de maio de 2020.

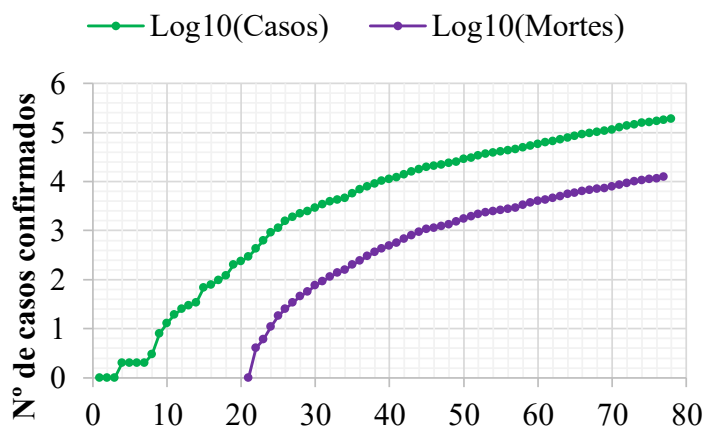

Fonte: Os autores.

Dias de contaminação

O Professor pode, também, utilizar dados locais e explorar a compreensão dos gráficos como na Figura 8, que apresenta o número de casos projetados (sem isolamento social) e dados reais do Estado da Bahia, em frequência acumulada nos vinte primeiros dias da pandemia, contados a partir do primeiro caso notificado. Pode explorar, baseado em Curcio (1989) e Monteiro (2006), por exemplo, diferentes tipos de leitura: quais foram os valores dos números projetado e real em 25 de março? (leitura dos dados). Qual é a diferença entre esses valores? (leitura entre os dados). Para a leitura além dos dados, o professor pode solicitar aos estudantes que projetem o número de casos confirmados para os dois próximos dias e para 30 dias depois. Além disso, pode instigar os estudantes a levantar hipóteses e, posteriormente, comparar com os dados reais. Esta questão pressupõe que nas justificativas os estudantes articulem suas opiniões e crenças sobre a Covid-19, baseadas nos dados apresentados (senso crítico). Estas questões também podem contribuir para o Letramento Estatístico (Gal, 2002) visando o desenvolvimento da postura crítica. Para tal, o professor pode realizar uma pesquisa para investigar a percepção dos estudantes sobre a doença e hábitos
Casos acumulados de COVID-19 por data de notificação $\swarrow$

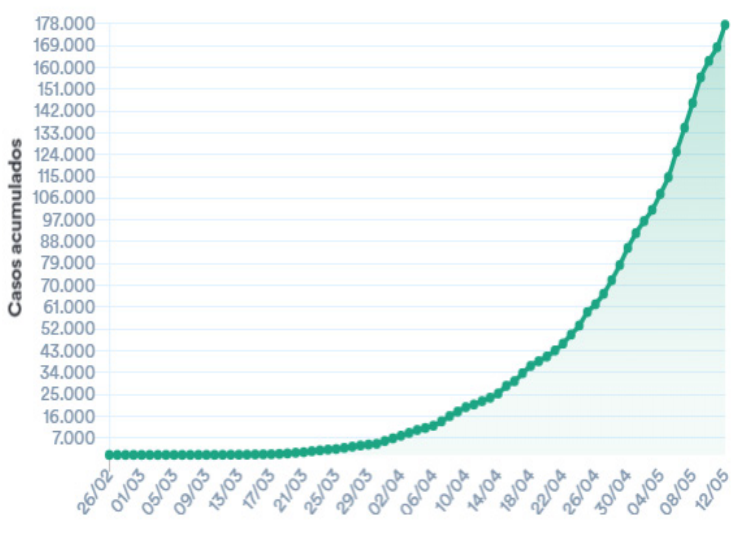

Data da notificaçăo

assumidos diante da pandemia, o perfil da população em torno da escola, condições sanitárias (se o bairro tem água encanada e esgoto), condição socioeconômica, acesso à saúde, dentre outras características que podem impactar o avanço da pandemia.

Figura 8 - Casos projetados e reais da Covid-19 na Bahia

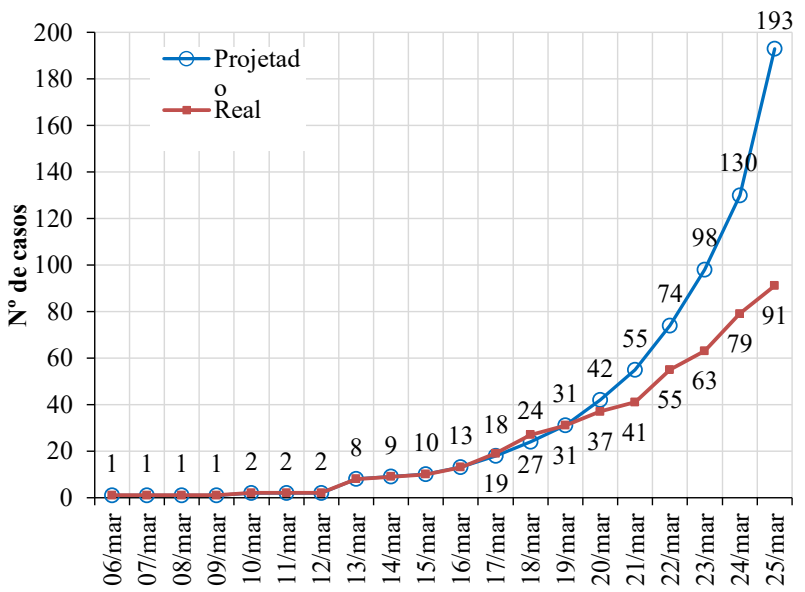

Fonte: Baseado em Secretaria de Saúde da Bahia - SESAB.

\subsection{Caso de Ensino 5: Crescimento exponencial em contexto}

Na compreensão de como se dá a velocidade de transmissão da doença, ou seja, como os novos casos confirmados estão surgindo, o professor pode descrever a curva de crescimento destes números usando modelos matemáticos. Com isso, é possível fazer previsões de número de casos confirmados e conhecer o número de casos esperados para qualquer momento do tempo. Por exemplo, no caso do Covid-19, a curva de crescimento do número de novos casos é considerada uma curva de crescimento exponencial pois, inicialmente, os casos surgem de forma lenta e rapidamente cresce. A taxa de contágio é definida como o número médio de pessoas contagiadas por uma pessoa infectada, isto é, quantos casos 
novos surgem a partir de uma pessoa infectada.

Para compreender de forma bastante intuitiva como funciona o crescimento exponencial, podemos utilizar o vídeo de Mauricio Féo (Figura 9), que supõe um lago que está sendo tomado por Vitórias-Régias (VR) e que a cada tempo multiplica (duplica, triplica, quadruplica etc.). A pergunta que o autor faz é: qual é o último dia que devemos limpar o lago removendo as VR para evitar que o lago seja completamente tomado pela planta? Isso depende da capacidade de reprodução das VR e da capacidade de remoção.

Esta situação pode ser trabalhada em sala de aula, já que o professor pode utilizar um espaço em cartolina ou papel metro e, com tampinhas de garrafa, representar as VR. Assim, um grupo trabalharia com uma taxa de crescimento igual a dois, outro grupo com uma taxa igual a três e assim por diante. Também, é interessante ter um grupo que trabalhe com crescimento linear. O professor pode sugerir aos estudantes a construção de tabelas e gráficos para acompanhar o crescimento das VR em cada situação.

Figura 9 - Coronavírus e Vitórias-Régias: Entendendo a curva exponencial do Coronavírus
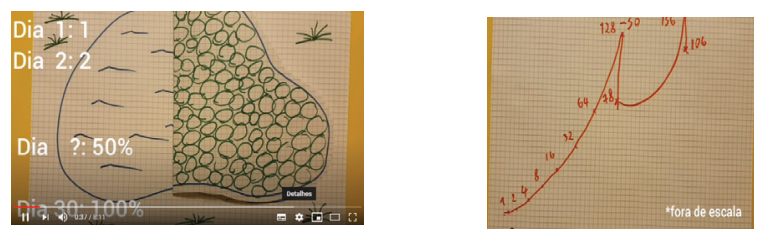

Fonte: Mauricio Féo, https://www.youtube.com/watch?v=s-lgS-4Xqy0.

Neste caso, o professor deve ter o cuidado de distinguir a variável número de VR e número de VR acumulado. Por exemplo, se a taxa de reprodução for dois, o número de VR passa de 1, para 2, para 4 e assim sucessivamente. Todavia, o número de VR acumulado passa de 1 para 3 , para 7 e assim sucessivamente. Desta forma que é contado o "número de casos da Covid-19".

\subsection{Caso de Ensino 6: Análise do crescimento exponencial no software Geogebra}

O Professor também pode realizar esta experimentação utilizando aplicativos matemáticos como o Geogebra que, segundo Bortolossi (2016), é um software gratuito de muita aceitação pelos professores de Matemática e comumente adotado nos cursos de formação de professores de Matemática. Como ele permite criação de cenários, o professor pode propor situações de investigação adequando a função exponencial de acordo com a situação da pandemia na cidade, estado ou região da escola. Para o autor, uma das potencialidades deste software é a possibilidade de mudar os elementos iniciais de uma construção de forma que o estudante possa, em tempo real, verificar como estas mudanças afetam os resultados, o que auxilia na compreensão dos conceitos e no papel de cada parâmetro na construção do modelo.

Neste artigo propomos duas construções no Geogebra, que são chamadas de applets, as quais ilustram situações apresentadas e as disponibilizamos para que os professores possam se apropriar e, na medida do possível, trabalhar com os estudantes.

Na construção ilustrada na Figura 10 podemos modificar dois parâmetros, a saber: o valor de $\mathrm{k}$, o qual indica o número de infectados no primeiro dia, e o valor do parâmetro "a", o qual representa em quantos dias o número de casos dobra em uma determinada localidade, e assim observar a relação entre estes dois parâmetros.

Neste applet também é possível analisar a velocidade de crescimento de uma função exponencial, geralmente considerada muito abstrata para alunos do Ensino Médio, e que, com o software, fica mais dinâmico. Para tal, o professor pode sugerir aos estudantes moverem o ponto A e observarem as alterações em suas imagens, os pontos $\mathrm{C}$ (exponencial) e D (linear). Outras abordagens ainda são possíveis de serem propostas, como a restrição ao domínio, tendo em vista que neste contexto está sendo considerada a variável tempo, o que inviabiliza valores negativos. O professor ainda pode mencionar a interseção de funções, o que este applet também permite explorar.

Figura 10 - Tela do Applet para simular o crescimento exponencial da Covid-19.

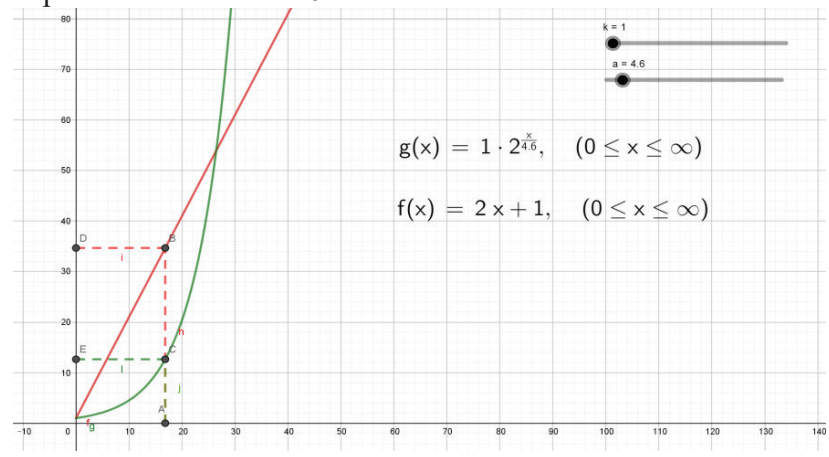

Fonte: Construção dos autores, disponível em: https://www.geogebra. org $/ \mathrm{m} / \mathrm{c} 5 \mathrm{jcwbdh}$.

O outro applet que propomos que o professor utilize com seus estudantes tem por objetivo reproduzir o modelo de velocidade de contágio da Covid-19 e ressaltar a importância do isolamento social defendido por pesquisadores e epidemiologistas para alterarmos a curva de crescimento da doença. Neste applet, ao clicar em uma pessoa (Figura 11a), o professor está informando para o software que aquele indivíduo aderiu ao isolamento e permaneceu em casa e, com isso, não infectaria outras três pessoas. Na simulação da Figura 11a destacamos em azul e em vermelho duas pessoas para permanecerem em suas casas e, com isto, elas não colaborariam com o crescimento do contágio e, consequentemente, as pessoas destacadas em verde não correriam o risco de serem contaminadas por essas pessoas, como ilustrado na Figura 11b. 
Figura 11 - Efeitos da mitigação no Geogebra

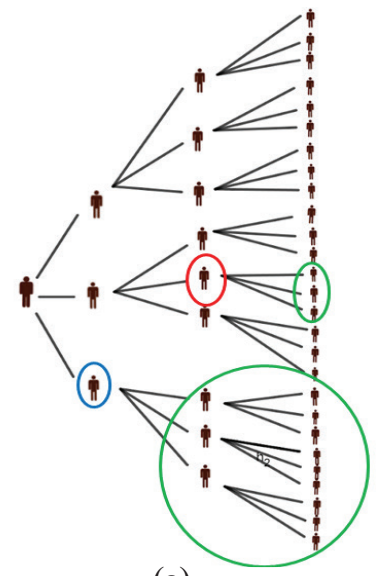

(a)

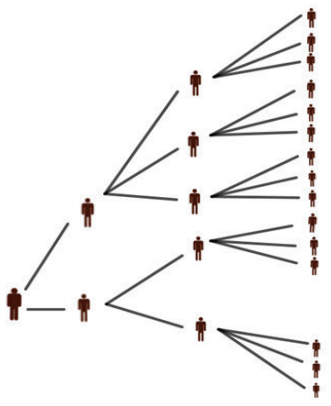

(b)
Fonte: Baseado em https://www.geogebra.org/m/mqyftquy.

Portanto, ao utilizar estes applets, o professor possibilita aos estudantes refletir e compreender a importância de acompanharem as orientações dos pesquisadores de todo o mundo que defendem o isolamento social horizontal como a principal medida para conter o avanço da Covid-19.

Os seis Casos de Ensino apresentados nesta seção têm por intenção ampliar tanto o conhecimento do conteúdo específico e pedagógico dos professores sobre os conceitos matemáticos e estatísticos envolvidos no contexto da pandemia da Covid-19, bem como o conhecimento curricular por meio do trabalho interdisciplinar que o contexto da pandemia da Covid-19 possibilita. A variedade de abordagens e representações aqui apresentas pode auxiliar os professores tanto a atender as diferentes formas de aprender dos estudantes como superar as dificuldades que estes encontram na compreensão dos conceitos matemáticos e estatísticos. Isto pode ser alcançado seja com material concreto, como sugerido no Caso de Ensino 5, que envolve a simulação de crescimento das VR no lago com o uso da tecnologia digital, como no Caso de Ensino 6, com os applets de simulação do Geogebra, por meio de representações gráficas, entre outros recursos aqui apresentados.

\section{Considerações Finais}

Neste artigo evidenciamos os conhecimentos necessários para uma ação pedagógica que torne os conceitos matemáticos e estatísticos compreensíveis aos estudantes. O conhecimento pedagógico do conteúdo é fundamental, uma vez que a forma como o professor seleciona e organiza o material didático para ensinar os conceitos de matemática e estatística evidencia seu conhecimento do conteúdo e o conhecimento curricular. O conhecimento dos conteúdos a serem trabalhados e o conhecimento da forma como o currículo escolar está estruturado, tanto na sua disciplina quanto nas demais, possibilita ao professor trabalhar na direção de uma organização interdisciplinar que atenda às demandas atuais da sociedade e permita aos estudantes tomar consciência de suas possibilidades enquanto cidadãos.
Os dados gerados pela pandemia da Covid-19 proporcionam uma oportunidade ímpar para o professor da Educação Básica contextualizar os conceitos de Estatística, focando na importância do letramento estatístico, do conhecimento matemático e da leitura dos dados, bem como a capacidade de analisar e interpretar estes de forma crítica, mas ressaltando que isto pode gerar sentimentos e emoções devido à morte de pessoas próximas, por exemplo.

Para conscientizar as pessoas sobre a importância do distanciamento social e "achatamento da curva" é preciso promover a compreensão de conceitos de Epidemiologia, Matemática e Estatística. Compreender como os dados são gerados, saber ler e interpretar gráficos e entender o processo de modelagem de variáveis estatísticas é fundamental para promover o letramento estatístico e o senso crítico dos estudantes. Com este entendimento, procuramos evidenciar neste artigo, por meio de seis Casos de Ensino, como o professor pode trabalhar em contexto a fim de conscientizar os estudantes de forma a fazer uma leitura crítica e tomar decisões com base em princípios éticos, solidários em relação ao cuidado de si mesmo, dos outros e do planeta, como preconizado na $\mathrm{BNCC}$.

\section{Referências}

Arteaga, P. (2011). Evaluación de conocimientos sobre gráficos estadísticos y conocimientos didácticos de futuros profesores. Tese de Doutorado. Universidad de Granada, Granada.

Bortolossi, H. J. (2016). O uso do software gratuito GeoGebra no ensino e na aprendizagem de estatística e probabilidade. Vidya, 36(2), 429-440.

Brasil (2002). Profissionalização de auxiliares de enfermagem: cadernos do aluno: saúde coletiva. Ministério da Saúde. Secretaria de Gestão e Investimento em Saúde. Projeto de Profissionalização dos Trabalhadores da Área de Enfermagem. Brasília: MS.

Brasil (2018). Base Nacional Comum Curricular. Ministério de Educação. Brasília: MEC.

Brasil (2019). Diretrizes Curriculares Nacionais para a Formação Inicial de Professores para a Educação Básica - Base Nacional Comum para a Formação Inicial de Professores da Educação Básica (BNC-Formação). Ministério de Educação. Brasília: MEC.

Castells, M. (2003). A galáxia da internet: reflexões sobre a internet, os negócios e a sociedade. Rio de Janeiro: Jorge Zahar.

Cazorla, I. M. \& Castro, F. C. (2008). O papel da Estatística na leitura do mundo: o letramento estatístico. Publicatio UEPG: Ciências Humanas, Ciências Sociais Aplicadas, Linguística, Letras e Artes, 16(1), 45-53.

Cobb, G. W. \& Moore, D. S. (1997). Mathematics, statistics, and teaching. The American Mathematical Monthly, 104(9), $801-823$

Curcio, F. R. (1989). Developing graph comprehension. Virginia: National Council of Teachers of Mathematics.

D’Ambrosio, U. (2005). Armadilha da mesmice em educação matemática. Bolema, 18(24), 95-109.

Diniz, L. N. \& Borba, M. C. (2012). Leitura e interpretação de 
dados prontos em um ambiente de modelagem e tecnologias digitais: o mosaico em movimento. Bolema, 26(43), 163-190.

Gal, I. (2002). Adults' statistical literacy: Meanings, components, responsibilities. International Statistical Review, 70(1), 1-25.

Garfield, J. \& Ben-Zvi, D. (2008). Developing students'statistical reasoning: Connecting research and teaching practice. New York: Springer.

Lopes, C. E. (2008). O ensino da estatística e da probabilidade na educação básica e a formação dos professores. Cad. Cedes, Campinas, 28(74), 57-73.

Lopes, C. E. (2013). Educação Estatística no Curso de Licenciatura em Matemática. Bolema, Rio Claro (SP), 27(47), 901-915.

Miola, A. F. S. \& Pereira, P. S. (2012). Uma análise de reflexões e de conhecimentos construídos e mobilizados por um grupo de professores no ensino de números decimais para o sexto ano do Ensino Fundamental. Práxis Educativa, 7(2), 533-558.

Monteiro, C. E. F. (2006). Explorando a complexidade da interpretação de gráficos entre professores em formação inicial. Cadernos de Estudos Sociais, 22(2), 211-224.

Monteiro, C. E. F. \& Selva, A. C. (2001). Investigando a atividade de interpretação de gráficos entre professores do ensino fundamental. 24a Reunião Anual da Associação Nacional de Pesquisa em Educação - ANPED (pp. 1-16). Caxambu: ANPED.
Oliveira, J. P. (2018). Aprendizagem em Estatística numa perspectiva transdisciplinar: uma possibilidade? Dissertação de Mestrado. Programa de Pós-graduação em Educação Matemática. Universidade Estadual de Santa Cruz. IlhéusBA.

Shulman, L. S. (1986). Those who understand: Knowledge growth in teaching. Educational Researcher, 15(2), 4-14.

Shulman, L. S. (1987). Knowledge and teaching: Foundation of a new reform. Harvard Education Review, 57(1), 1987.

Shulman, L. S. (2014). Conhecimento e ensino: Fundamentos para a nova reforma. Cadernos Cenpec, 4(2), 196-229.

Silva, A. F. G., Alves, T. A. S., Pietropaolo, R. C. \& Amorim, M. E. (2020). Propriedades da Média: um Estudo sobre respostas dadas por professores para Casos de Ensino. Educação Matemática em Revista, 25(66), 184-200.

Skovsmose, O. (2007). Educação crítica: incerteza, matemática e responsabilidade. (Traduzido por Maria Aparecida Viggiani Bicudo). São Paulo: Cortez.

Torre (2004). Aprendizagem integrada: uma aprendizagem para a vida. In: Moraes, M. C.; Torre, S. Sentir pensar: fundamentos e estratégias para reencantar a educação. Petrópolis: Vozes.

Zieffler, A., Garfield, J. \& Fry, E. (2018). What Is Statistics Education? In D. Ben-Zvi et al. (eds.), International Handbook of Research in Statistics Education, Springer International Handbooks of Education, Chapter 1, 37-69. 